\title{
Estampa y aportaciones de tres maestros renovadores: Norberto Hernanz, Pablo de Andrés y David Bayón
}

Rosa Ortiz de Santos. Universidad de Valladolid

Recepción: 28.02.2018 | Aceptado: 5.03.2018

Correspondencia a través de ORCID: Rosa Ortiz de Santos

iD 0000-0003-2408-1645

Citar: Ortiz de Santos, R. (2018). Estampa y aportaciones de tres maestros renovadores: Norberto Hernanz, Pablo de Andrés y David Bayón. ReiDoCrea, 7, 63-78.

Resumen: El primer tercio del siglo XX conoce la cimentación de la renovación educativa en España, especialmente en el periodo que comprende la Segunda República, y más específicamente, durante el bienio progresista. Los maestros y las maestras adquieren un papel esencial en el proceso de renovación pedagógica y de mejora de las escuelas. Desde esta aportación se pretende recuperar la figura y la trayectoria individual y colectiva de tres maestros renovadores cuyas iniciativas tuvieron una gran influencia en la educación de su época: Norberto Hernanz Hernanz, Pablo de Andrés Cobos y David Bayón Carretero. El estudio se ha llevado a cabo mediante una metodología cualitativa crítica guiada por el proceso de investigación histórico-educativa y abordada mediante la técnica de análisis documental de fuentes primarias y secundarias de diferente procedencia y orden. Las categorías de análisis nos han permitido construir un breve relato sobre cada uno de los maestros y establecer las iniciativas y los proyectos que colectivamente llevaron a cabo. No cabe duda de que los tres maestros son un ejemplo del sector más renovador del magisterio que contribuyó a mejorar la educación, apoyando activamente la reforma educativa que acometió la Segunda República en España.

Palabras clave: Historia de la Educación | Docente de Escuela Primaria

Presentation and Contributions of Three Renovating Teachers: Norberto Hernanz, Pablo de Andrés and David Bayón

Abstract: The first third of the twentieth century saw the foundations of educational renovation in Spain, especially in the period comprising the Second Republic, and more specifically, during the progressive biennium. Teachers acquire an essential role in the process of pedagogical renewal and improvement of schools. This contribution aims to recover the figure and individual and collective career of three renovating teachers whose initiatives had a great influence on the education of their time: Norberto Hernanz Hernanz, Pablo de Andrés Cobos and David Bayón Carretero. The study was carried out using a critical qualitative methodology guided by the process of historical-educational research and approached through the technique of documentary analysis of primary and secondary sources of different origin and order. The categories of analysis have allowed us to build a short story about each one of the teachers and establish the initiatives and projects that they collectively carried out. There is no doubt that the three teachers are an example of the most innovative sector of teaching that contributed to improving education, actively supporting the educational reform undertaken by the Second Republic in Spain.

Keywords: History of Education | Primary School Teachers

Agradecimientos: Transmitimos nuestro agradecimiento a la familia de Norberto Hernanz Hernanz, y más específicamente a su nieto, Norberto García Hernanz, con quien establecimos contacto y quien nos cedió algunos documentos, aún inéditos, que nos han aportado mucha información para poder construir los relatos. De igual modo, agradecemos a Ainhoa Zufriategui Capurro haber compartido la excelente biografía sobre Pablo de Andrés Cobos, documento básico para el desarrollo de este trabajo.

\section{Introducción}

El 14 de abril de 1931 se proclamó la Segunda República en España; para muchos, esta nueva forma de gobierno venía acompañada de una valiosa oportunidad de regeneración, de progreso y de mejora con respecto a la situación fuertemente desesperanzadora heredada de la Dictadura de Miguel Primo de Rivera (Martínez, 
2000). En este nuevo escenario se impulsaron numerosos proyectos y reformas que constituirían el inicio de un periodo de cambios importantes en la sociedad.

En materia educativa las reformas que se llevaron a cabo fueron cuantiosas; se prestó atención a la construcción de escuelas, a la mejora de los centros escolares rurales, al establecimiento de una escuela laica ajustada a la nueva situación del Estado, al sistema de inspección educativa y a la creación de iniciativas pedagógicas encaminadas a la mejora de la calidad de la educación y a lograr una mayor atención a toda la población en edad escolar. Sin menoscabo de lo anterior, algunos de los proyectos más importantes estuvieron orientados a mejorar la formación y las condiciones del magisterio español (Puelles, 2011). No es casualidad que el entonces ministro de Instrucción Pública, Marcelino Domingo, calificara a este periodo como "La República de los maestros", pues existía la certeza de que eran los docentes, junto con el poder educativo de la escuela, los principales agentes a través de los cuales se podía lograr una transformación eficaz de la sociedad (Martín, 2015). Rodolfo Llopis ${ }^{1}$ incide en la importancia que tiene el profesorado de primera enseñanza dentro de este proceso, afirmando que "el maestro es el alma de la escuela" (Llopis, 1933-2005, p. 86), definición que anteriormente hizo el docente Bernardo Miguel Mancebo en Escuelas de España (julio, 1930); asimismo, el pedagogo Manuel Bartolomé Cossío describía a los docentes como "libertad y espíritu" (Cossío, 1929, p. 6) y Norberto Hernanz Hernanz apuntaba que las cualidades de un buen maestro eran "vocación, ciencia y talento" (Hernanz, 1929, p. 15).

Nos encontramos en un momento de especial renovación educativa, caracterizado por una latente y cada vez más reconocida riqueza pedagógica, que tuvo lugar gracias a las inquietudes y a la actividad de maestros y maestras y de otros profesionales e instituciones preocupadas por la mejora de la educación. Desde numerosas aportaciones se viene denominando a los docentes de primera enseñanza de este periodo "misioneros laicos" (Martín, 2015) o "maestros republicanos". Según Pozo (2012) este último término engloba a los maestros de tres generaciones diferentes (Figura 1) que tienen como objetivo común y prioritario lograr consolidar una educación nueva mediante la construcción de una "Escuela Nueva" que necesariamente deberá ser pública para, a través de ella, llegar a transformar el contexto español.

Magisterio
regeneracionista

Figura 1. Los maestros republicanos. Fuente: elaboración propia a partir de Pozo (2012)

\footnotetext{
${ }^{1}$ Director General de Primera Enseñanza
} 
Estos tres grupos de maestros y maestras convivieron en un mismo periodo temporal pero, tal y como expone Pozo (2012), pertenecían a generaciones diferentes. Formaban parte del magisterio regeneracionista algunas de las figuras señeras de la educación, como los maestros Ángel Llorca i García y Félix Martí Alpera; era un grupo fuertemente influenciado por las corrientes educativas europeas que descubrieron en los viajes pedagógicos que realizaron en grupo o individualmente (Marín, 1991), pensionados por la Junta para la Ampliación de Estudios e Investigaciones Científicas (en adelante, JAE). Precisamente, este fue el punto de convergencia que unía a este colectivo y al magisterio renovador: la fuerte influencia que sobre ellos tuvo el movimiento de la Escuela Nueva. De esta otra generación formaron parte un gran número de entusiastas maestros y maestras de todos los rincones de España; por su repercusión y su trascendencia en la investigación histórico-educativa mencionamos a Luis Huerta Naves, a Concepción Sáinz-Amor Alonso, a María Sánchez Arbós o al aragonés Santiago Hernández Ruiz. Situamos en este grupo generacional a los maestros segovianos Norberto Hernanz Hernanz, Pablo de Andrés Cobos y David Bayón Carretero, los protagonistas de esta aportación. Por su parte, el magisterio nuevo integraba a docentes que terminaron sus estudios ya comenzada la Segunda República. Fueron caracterizados por su optimismo y su afán en aplicar los métodos que habían aprendido y heredado de los maestros de generaciones anteriores; Manuela García Trapero o Pilar Montero Hernández fueron algunas de las maestras que pertenecieron a esta generación.

No cabe duda de que "la República consiguió obtener un numeroso grupo de maestros y maestras poseedores de unas magníficas dotes pedagógicas, y entregados con pasión y amor a su quehacer" (Martín, 2015, p. 152). Así lo evidencian las investigaciones existentes sobre la concepción y la relevancia de la figura del maestro dentro de este periodo. Molero (1997) explica las vicisitudes de las reformas que se acometieron sobre los maestros y las escuelas, permitiéndonos conocer el perfil profesional de los docentes republicanos y haciendo hincapié en la importancia de su labor; esto mismo hace Navarro (2002) tomando como referencia la figura de Rodolfo Llopis, importante impulsor de estos proyectos. Hernández (2007), por su parte, analiza la situación de los maestros durante la transición a la democracia y evidencia el reconocimiento que ha de tener la renovación pedagógica impulsada durante el bienio progresista de la Segunda República, del mismo modo que Martín (2015) emplea la técnica de análisis documental para darnos a conocer la profesión docente en dos periodos diferentes: la Segunda República y la posterior Dictadura.

Vicente (2006) insiste en que uno de los objetivos prioritarios era hacer de la sociedad una verdadera democracia, y en que en este contexto los maestros y las maestras "fueron un elemento fundamental en la difusión de los valores cívicos y democráticos que impulsaba la República" (p. 7). Por ello es necesario el reconocimiento de los mismos, que da lugar a aportaciones como el libro de Iglesias (2006), quien pretende reconocer el trabajo de los maestros republicanos mediante la reconstrucción de diez historias a partir de diferentes testimonios, o el artículo de Montanyà (2006), quien homenajea a los docentes de las escuelas republicanas en Cataluña, que trabajaron con miras a lograr una eficaz transformación social partiendo de unos sólidos principios de igualdad. Asimismo, cada vez es más común encontrar trabajos de investigación dedicados a reconocer la excelente labor de las maestras republicanas, dándolas visibilidad y contribuyendo a la construcción de la historia de las mujeres en España. Destacamos a Sánchez (2010), que define a las maestras rurales en el contexto republicano, o la conocida obra Las maestras de la República (2012), en la que colaboran numerosas profesionales de la investigación en historia de la educación. 
Además de estos estudios, existen otros orientados a presentar la biografía y la obra pedagógica y educativa de maestros y maestras concretos. Desde diferentes lugares de España se está llevando a cabo la ardua tarea de recopilar información sobre los profesionales más relevantes en la educación republicana. Contamos con los recientes trabajos de Juan (2017) y de Agulló y Romans (2018); el primero recoge información sobre los profesionales más representativos de la educación en Aragón, mientras que los segundos coordinan una obra destinada a recuperar la memoria histórica educativa de la Comunidad Valenciana mediante la presentación de las trayectorias y las prácticas innovadoras de los maestros y de las maestras de esta comunidad durante el primer tercio del siglo XX. En el caso de Segovia, la Real Academia de Historia y Arte de San Quirce está trabajando en la elaboración de un Diccionario Biográfico de Personalidades (1900-1936) con motivo del centenario de la Universidad Popular Segoviana que tendrá lugar en 2019; en la relación de biografiados se observan los nombres de los tres maestros que presentamos en este artículo, o los de otros docentes -Mariano Domínguez Martín y Pedro Natalías García- e intelectuales como el inspector de primera enseñanza Antonio Ballesteros Usano o el editor de prensa Carlos Martín Crespo. Este trabajo podría complementarse con el de Dueñas y Grimau (2004), donde se recoge un listado de 243 maestros y maestras segovianos que fueron sancionados por la represión franquista. Además de estos ejemplos, Marín $(1990,1991)$ recoge información sobre los docentes pensionados por la JAE.

Existen innumerables aportaciones a coloquios, congresos y jornadas científicas sobre diferentes maestros y maestras, así como tesis doctorales y capítulos de libro; no obstante, es preciso dirigir la mirada hacia la producción científica que se ha realizado mediante artículos de investigación. Jiménez (1992) y Moreno (2005) analizan la contribución a la renovación pedagógica de Santiago Hernández Ruiz y Félix Martí Alpera respectivamente; los escritos de Juan (1999), González (2009) y Jimeno (2009) nos acercan a la figura de María Sánchez Arbós y la aportación de Lázaro (2009), al maestro Luis Huerta Naves, de igual modo que Vilafranca y Vilanou (2001) y Alonso (2009) recopilan información sobre Concepción Sáinz-Amor. Son numerosos, además, los trabajos sobre el elenco de maestros y maestras que conformaban el Grupo Escolar Cervantes de Madrid, tanto de su director Ángel Llorca (Pozo, 1987; López, 1995), como de las excelentes maestras Elisa López Velasco (Cuenca, 2003) y Justa Freire Méndez, de quien es preciso destacar el libro biográfico de Pozo (2013).

Contamos, por tanto, con un amplio marco de producción científica que da sentido y orienta nuestro estudio. El objetivo prioritario de esta aportación es recuperar la figura de los maestros Norberto Hernanz Hernanz, David Bayón Carretero y Pablo de Andrés Cobos. Estos maestros no solo contribuyeron a renovar el panorama educativo segoviano, sino que su relevancia es mucho mayor: son autores de importantes iniciativas educativas que tuvieron una gran influencia en la educación de su época.

\section{Métodos}

\section{Fenómeno estudiado, diseño y procedimiento de investigación}

Podríamos etiquetar esta investigación como un estudio cualitativo. Partiendo de su perspectiva histórica, se han tomado como referencia las fases del modelo de investigación histórico-educativa (Figura 2) que han marcado el proceso a seguir. 


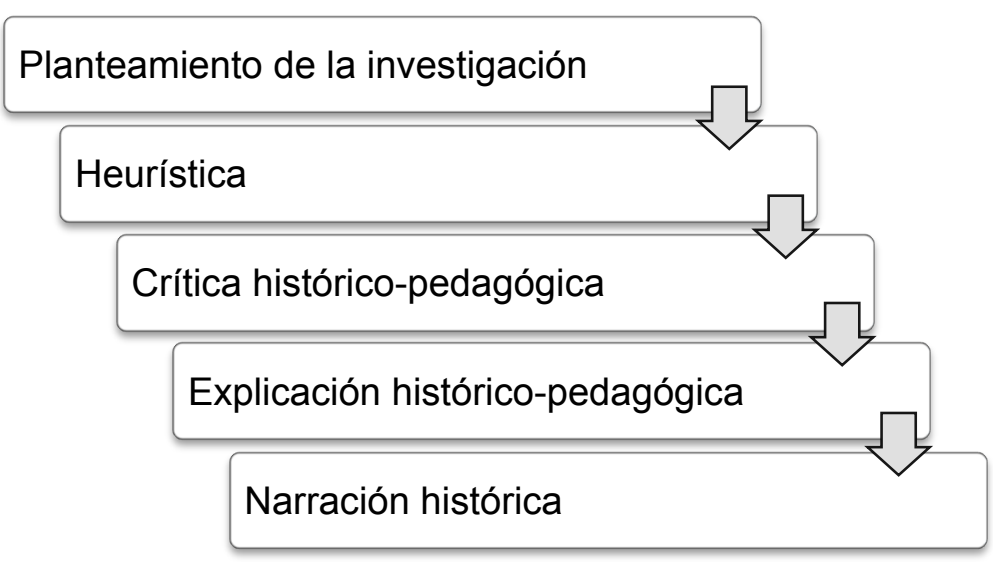

Figura 2. Fases de la investigación. Fuente: elaboración propia a partir de las fases de Ruiz (1976), reformuladas por Tiana (1988) y Ruiz (1997).

1. La primera fase implica, necesariamente, la delimitación del tema o fenómeno de estudio. En esta aportación se pretende recopilar y recuperar información relevante que nos ayude a realizar una breve presentación de tres maestros del magisterio renovador de la Segunda República Española: Norberto Hernanz, David Bayón y Pablo de Andrés, cuyo conocimiento es esencial en los procesos de reconstrucción de la historia de la renovación pedagógica en el contexto español.

2. La heurística es la selección de las fuentes de análisis. Los documentos consultados han sido cuantiosos y de diferente naturaleza: (a) Artículos y trabajos científicos que nombran o nos dan pistas sobre alguno o varios de nuestros protagonistas (Hermida, 2005; Andrés y Mora, 2011; Mora y Hermida, 2011; Ortiz, 2017). (b) Artículos en prensa actual y recursos electrónicos (García, 2008; Estebaranz, 1, marzo, 2016). (c) Libros con información sobre Pablo de Andrés Cobos (Andrés, Zufriategui y Estebaranz, 2017² y Andrés, Zufriategui y Estebaranz, $2015^{3}$ ). (d) Testimonios escritos y documentos inéditos cedidos por la familia de Norberto Hernanz -Memorias de Norberto Hernanz (Hernanz, s.f.) y un documento escrito por su hijo, Máximo Hernanz Mínguez (Hernanz, 2008)-. (e) Prensa de la época. (f) Artículos, libros y otros documentos de la época escritos por los maestros presentados. (g) Los ejemplares de la revista pedagógica que los tres docentes dirigieron, Escuelas de España, digitalizados en la Hemeroteca de la Biblioteca Nacional Española.

3. Los documentos anteriormente nombrados deben ser sometidos a la fase de crítica histórico-pedagógica para así comprobar su grado de validez mediante la crítica externa y la crítica interna, que a su vez conllevan otros procedimientos (Figura 3).

\footnotetext{
${ }^{2}$ Edición de la obra El maestro, la escuela y la aldea de Pablo de Andrés Cobos.

${ }^{3}$ Pequeño manual que forma parte de la edición conmemorativa de Estampas de Aldea.
} 


\section{Crítica externa}

- Crítica de procedencia

- Crítica de restitución

\section{Crítica interna}

- Crítica de interpretación

- Crítica de competencia

- Crítica de sinceridad

- Crítica de exactitud

-Verificación de testimonios

Figura 3. Crítica histórico-pedagógica. Fuente: elaboración propia a partir de las fases de Ruiz (1976), reformuladas por Tiana (1988) y Ruiz (1997).

Para responder a la crítica externa se ha procurado conocer aspectos generales de las fuentes documentales, como por ejemplo, el origen, la fecha de publicación o la autoría. Asimismo, atendiendo a la crítica de restitución se han consultado la mayor parte de los documentos de carácter histórico en su formato original. Entre ellos, 12 ejemplares de Escuelas de España ${ }^{4}$, Los clásicos en la escuela ${ }^{5}$ (1935), de Norberto Hernanz, así como Juicios y Figuras $^{6}(1969)$ y la memoria de los viajes de estudios de Pablo de Andrés Cobos, Un viaje por las escuelas de España ${ }^{7}$ (1927).

En lo que respecta a la crítica interna, se han utilizado varios procedimientos que nos dan la certeza de que la información que se recoge acerca de los maestros en cada uno de los documentos se corresponde con la realidad de los hechos (Tiana, 1988). La recopilación y el estudio de documentos escritos por los propios protagonistas, el trabajo realizado por parte de otros profesionales y los propios testimonios de familiares de los maestros, nos permite establecer un vínculo más directo con los docentes, favoreciendo una mejor interpretación de los datos. De este modo, también vemos abordada la crítica de competencia, siendo los propios maestros, sus familiares o personas con una excelente formación histórico-educativa, las mejores fuentes para llevar a cabo la tarea de evocar y presentar a Hernanz, Bayón y Cobos $^{8}$. Por lo tanto, esta triangulación de la información, junto con el estudio del contexto sociohistórico, nos permite dar respuesta a la crítica de sinceridad y de exactitud, así como llevar a cabo una adecuada verificación de los testimonios.

4. Esta fase hace referencia al análisis. Se refiere a la interpretación y posterior redacción de la información, para lo cual es fundamental conocer el periodo en el que se encuadra el proyecto. Esta fase ha sido trabajada conjuntamente con la "narración histórica", orientada a la exposición y publicación de los

\footnotetext{
${ }^{4}$ Cinco ejemplares propiedad de Norberto García Hernanz, nieto de Norberto Hernanz (enero, abril y julio de 1929, julio de 1931 y enero de 1932), un ejemplar del depósito de la Biblioteca Pública de Segovia (julio, 1930) y seis ejemplares del Archivo de la Diputación Provincial de Segovia (abril de 1930, enero de 1931 y enero, febrero, marzo y abril de 1934).

${ }^{5}$ Cedido por Norberto García Hernanz.

${ }^{6}$ Conservado en la Facultad de Filosofía y Letras de la Universidad de Valladolid.

${ }^{7}$ Conservado en el Archivo de la Diputación Provincial de Segovia.

${ }^{8} \mathrm{Se}$ utiliza el segundo apellido ya que es el que comúnmente aparece en los escritos cuando se hacen referencias continuas al maestro de escuela.
} 
resultados de la investigación. Dicha exposición se recoge en el apartado de "resultados" y en el de "discusión", en el cual encontramos, además, las conclusiones del trabajo. De igual modo, la difusión de los resultados se satisface mediante la publicación del trabajo en forma de artículo científico.

\section{Técnica de recogida de información: análisis documental}

La investigación se aborda mediante la técnica de análisis documental de las fuentes nombradas en líneas anteriores. Contamos, por tanto, con documentos escritos (MacDonald y Tipton, 1993) que, en su mayor parte, constituyen textos pedagógicos dentro de la categoría de documentos institucionales (Corbetta, 2003). Según los tipos de documentos histórico-pedagógicos (Tiana, 1988 y Ruiz, 1976), estamos hablando de fuentes primarias o directas -en los casos (d), (e), (f) y (g)- y de fuentes secundarias en los casos restantes.

\section{Instrumento de análisis de datos}

El análisis de la información se ha llevado a cabo mediante una lectura en profundidad de los documentos citados, con la finalidad de obtener los datos suficientes para construir la estampa de los maestros. Para ello, hemos definido unas categorías de análisis centradas en los siguientes tipos de información:

a) Datos biográficos: nombre y apellidos, fecha y lugar de nacimiento y defunción.

b) Formación y desarrollo profesional

c) Carácter renovador y contribuciones de cada uno al proceso de renovación pedagógica de la escuela y de la educación en España.

d) Aportaciones realizadas colectivamente por nuestros protagonistas.

e) Otros datos de interés

Bien es cierto que ha sido un esquema de análisis flexible y abierto, no completado en su totalidad en alguno de los casos.

\section{Resultados}

En este apartado presentamos a cada uno de los maestros estudiados.

\section{Norberto Hernanz Hernanz}

Norberto (Figura 4) nació el 8 de mayo de 1891 en un pequeño pueblo de la Segovia rural, Orejana; más concretamente, en el barrio de La Revilla. Allí pasó su infancia y ayudó a su familia en las labores del campo. Por todos era conocida la afición de Norberto a la lectura. Esta afición influyó en que su familia decidiese que en 1912, con la edad de 21 años, realizase el examen de ingreso en la Escuela de Magisterio de Segovia. Aprobó el examen; sus estudios en Segovia le permitieron aprender de las enseñanzas de Blas José Zambrano -el padre de la filósofa María Zambrano Alarcón, a quien consideraba un "verdadero maestro" (p. 70) ${ }^{9}$. Él mismo reflexiona acerca de la influencia que ejerció sobre su identidad profesional: "en las clases de D. Blas aprendí que el saber no consiste en recitar con soltura una retahíla de palabras

\footnotetext{
${ }^{9}$ Las citas literales del apartado destinado al maestro Norberto Hernanz han sido extraídas de sus Memorias (Hernanz, s.f.), pues es la fuente principal de la que emanan casi todas las demás fuentes consultadas para construir el relato de este maestro. El documento elaborado por Máximo Hernanz (Hernanz, 2008), también ha sido una fuente clave para comprender la sucesión de los acontecimientos.
} 
tomadas de un libro; sino el comprender, razonar y adquirir espíritu de observación y de crítica" (p. 70). En esta etapa también trabaría amistad con su compañero Lorenzo del Amo Sanz, procedente de Arcones (Segovia).

En 1914 se graduó y con 25 años aprobó las oposiciones. Optó por quedarse en la escuela unitaria de niños de Torre Val de San Pedro (Segovia), donde ejercería como maestro hasta 1929. Con la ingenuidad, además de la ilusión que acompaña a todo docente en sus primeras experiencias en la escuela, pudo comenzar a desarrollar numerosas iniciativas y prácticas pedagógicas. Aunque el edificio escolar tenía algunas limitaciones que no se solventaron -"los tablones del piso están bastante resquebrajados; las mesas son viejas e incómodas; las ventanas son más bien conatos..." (p. 80)- pese a los intentos de Norberto por llevar a cabo una reforma, el maestro fomentó la participación de la comunidad y de las familias mediante la realización de obras teatrales, favoreció el trabajo en grupo en el aula e impulsó los paseos escolares generando un mayor contacto con el entorno y procurando la aplicación de los contenidos educativos en el contexto real de los niños y de las niñas mediante una enseñanza práctica y activa. Para Norberto, se trataba de "hacer de la escuela una especie de taller" (p. 82); esto, además del desarrollo del pensamiento científico, crítico y libre del alumnado, se refleja en las prácticas que llevó a cabo.

En este periodo Norberto se veía a menudo con Lorenzo del Amo, quien había sido destinado en la escuela de La Salceda. Ambos maestros se reunían en el camino que unía los dos pueblos; para romper con el aislamiento al que estaban expuestos muchos maestros rurales, a Lorenzo del Amo se le ocurrió una idea: "¿Y si llamáramos a todos los compañeros del contorno y nos reuniéramos una vez al mes en uno de los pueblos?" (p. 4). Así es como, en 1921, nacerían los Centros, después denominados Centros de Colaboración Pedagógica (en adelante, CCP) que posteriormente, el inspector Antonio Ballesteros difundiría por la provincia. Esta iniciativa fue, además, una oportunidad de perfeccionamiento docente. Norberto, que ansiaba continuar formándose, ingresó en la Escuela Superior de Magisterio (Madrid); en este periodo asistió a algunas de las clases que Cossío u Ortega y Gasset impartían en el Museo Pedagógico. Asimismo, tuvo la oportunidad de conocer diferentes escuelas de España $^{10}$ (1927) gracias a las pensiones que la Diputación de Segovia concedía a los mejores maestros. Desde entonces, formó parte de una sucesión de iniciativas que compartirá con los otros dos maestros presentados, y que tratamos posteriormente.

Todas estas iniciativas, además de la difusión que empezaron a tener algunos de sus trabajos pedagógicos, le habían otorgado cierto prestigio, lo cual llegó a aturdir al que se consideraba "... un maestro rural que de pronto se ve metido en un ambiente ciudadano" (p. 125). Esto es así dado que en 1929 se trasladó a una escuela de Segovia, donde estuvo trabajando durante dos años, periodo en el que se crea el Partido Republicano en la ciudad -en el que militaban personas como Antonio Machado, Rubén Landa o Mariano Quintanilla-, del que Norberto es nombrado secretario. Los viajes que realizó a escuelas del extranjero ${ }^{11}$ en 1930 , de nuevo pensionado por la Diputación Provincial, le permitieron conocer los Centros de Interés de Decroly, que posteriormente ensayó en su escuela de Segovia. Continuó aplicando prácticas escolares pedagógicas, pues introdujo las excursiones al campo y a la ciudad, así como un pequeño laboratorio en el aula. Tal y como él expone, a estas actividades les faltó continuidad, debido a que en 1931 se trasladó a la escuela Baixeras tras conseguir una vacante del Patronato Escolar de Barcelona; fue maestro de enseñanza del castellano y utilizó la lectura como medio y base fundamental del trabajo en el aula. Fruto de ello nació Los clásicos en la escuela (1935). Los fines de

\footnotetext{
${ }^{10}$ Visitó con Bayón escuelas de Madrid, Zaragoza, Barcelona, Valencia, Granada y Huelva.

${ }^{11}$ Visitó escuelas de Francia, Bélgica y Suiza con Pablo de Andrés y David Bayón.
} 
semana aprovechaba para participar en varias "tertulias", una de maestros y de maestras, y otra a la que asistían inspectores de educación y profesores de la Normal.

Nunca abandonó esa inquietud y ese interés por seguir formándose, ni si quiera cuando la Guerra Civil Española sorprendió a Cataluña. En 1939 Norberto fue depurado de sus funciones y suspendido de empleo y sueldo, y hasta 1944, cuando se resolvió su expediente de depuración, mediante el cual era rehabilitado y destinado en Mayals (Lérida), montó un quiosco que no funcionó y trabajó en la Academia Lara de oposiciones. Norberto no quería trasladarse de Barcelona por lo que trabajó en un colegio de frailes en el que nunca se sintió cómodo, y posteriormente en un colegio del barrio de San Andrés, para terminar ejerciendo en la Academia Práctica de oposiciones hasta su jubilación. Norberto falleció en 1981.

\section{Pablo de Andrés Cobos}

Pablo (Figura 4) nació el 1 de junio de 1899 en La Cuesta (Segovia) ${ }^{12}$. Creció feliz en el entorno rural que siempre valoró y defendió, y al que estuvo vinculado durante toda su vida. A la edad de 16 años fue a estudiar a la Escuela Normal de maestros de Segovia con dos de sus hermanos, Clemente y Esteban. Allí, al igual que Norberto en años anteriores, tuvo de profesor a Blas J. Zambrano, a quien admiraba y quien le acercó a las tertulias presididas por Antonio Machado que se llevaban a cabo en el Café de la Unión, situado en la Calle Real de Segovia. Estos momentos de reunión y de intercambio intelectual fueron esenciales en su formación. De igual modo, fue una oportunidad para que entablase amistad con Mariano Quintanilla y el maestro Norberto Cerezo. El 12 de junio de 1917, a la edad de 18 años, se graduó en la Normal.

En 1920 aprobó la oposición para adquirir una plaza como maestro nacional. Le asignaron su primer destino en 1921, en Valdepeñas (Ciudad Real), donde trabajó hasta 1925. El 1 de septiembre de 1925 se trasladó como maestro a la escuela graduada de niños de La Granja de San Ildefonso (Segovia) y rápidamente tomó parte de las iniciativas que se estaban desarrollando en la provincia. En este nuevo contexto estableció una relación con la directora de la escuela graduada de niñas de la misma localidad, Enriqueta Castellanos Pereda, de la que se enamoró y con quien compartió su pasión por la buena educación.

En 1926 realizó un viaje por diferentes escuelas de España, pensionado por la Diputación Provincial de Segovia ${ }^{13}$. De esta experiencia nació su memoria Un viaje por las escuelas de España (1927). En 1929 volvió a ser pensionado para acudir a escuelas de países europeos. Estos viajes fueron esenciales en su formación, al igual que lo fueron las restantes experiencias que vivió, como la realización de una conferencia en el Ateneo de Madrid, a petición de Cossío (abril, 1930) ${ }^{14}$, o su

\footnotetext{
${ }^{12}$ Las fuentes principales en las que nos basamos para construir la estampa de Cobos son los trabajos de su biógrafa Ainhoa Zufriategui Capurro (Andrés, Zufriategui y Estebaranz, 2017 y Andrés, Zufriategui y Estebaranz, 2015), pues la presentación de la vida del maestro que ella expone bebe del resto de fuentes recogidas en la fase de "heurística", expuestas en el apartado de "metodología".

${ }^{13}$ Visitó escuelas de Madrid, Barcelona, Zaragoza, Valencia y Bilbao. Según las bases de las "pensiones para estudiantes pobres de la provincia" de 1925, además de Cobos, solicitaron la pensión para "estudios de ampliación" este mismo año los maestros María Fuencisla Moreno, Norberto Cerezo Marinero, Pedro Natalías, David Bayón, Juan Monje Cebrián, Lucía Lucha García y Ángel Gracia Morales; tras la valoración del tribunal, seleccionaron a Cobos y a Fuencisla Moreno (ADPSg, caja 4007).

${ }^{14}$ La petición se recoge en Juicios y Figuras (1970), en la "carta segunda" que Cossío envió a Cobos.
} 
nombramiento, un mes más tarde, como director de la escuela de la Granja en la que trabajaba. Durante los seis años que estuvo allí y junto a Enriqueta, impulsó la Sociedad Infantil siguiendo el modelo del grupo escolar Baixeras, y promovió otro tipo de actividades, como la organización de clases destinadas a personas adultas.

En febrero de 1931 se fue a Barcelona tras haber conseguido una vacante en el grupo Ramón Llull del Patronato Escolar. No obstante, es importante mencionar la asamblea que se celebra en verano de ese mismo año en La Granja, con la intención de atender a los intereses de la reforma educativa que iba a llevar a cabo la República. A esa asamblea a la que asiste Cobos, acuden unas veinte personas, según las conclusiones recogidas en Escuelas de España (Andrés, octubre, 1931); en ellas se defiende una escuela activa, única y laica, bajo los principios de libertad y prestando especial atención a las escuelas del ámbito rural.

En 1933 Cobos y Enriqueta abandonan Barcelona para trabajar en el Orfanato de El Pardo de Madrid, del que al final de ese mismo año se convierte en Director de Estudios hasta 1934, momento en el que presentan su dimisión; la experiencia vivida allí la recoge en Orfanato Nacional de El Pardo: tres meses de Director de Estudios. Durante este periodo también colaboró como misionero y como organizador y director de algunas de las Misiones Pedagógicas impulsadas por Cossío. En 1934 se incorporó como director en el grupo escolar Claudio Moyano (Madrid) hasta 1936; en 1935 publicó Estampas de aldea, que en 1937, en plena Guerra Civil Española, aparecería en el listado de libros prohibidos.

Cobos fue duramente represaliado; en 1936 fue acusado por "conspiración para la rebelión" e ingresó, en un primer momento, en la Cárcel Vieja de Segovia, siendo después trasladado a otras prisiones. En 1939 se resolvió su expediente, por el que fue suspendido de empleo y sueldo. A pesar de ello, nunca se separó de la docencia, y llegó a ejercer como maestro dentro de la prisión, hecho que hizo que redujesen su condena hasta octubre de 1940, cuando abandonó la cárcel. Cobos buscó diferentes medios para poder subsistir y salir adelante; trabajó como lechero y posteriormente en diferentes academias de oposiciones, llegando a fundar su propia academia Audiencia y la editorial Ancos. Esta es la editorial de sus obras Juicios y Figuras (1970) y Humorismo de Antonio Machado en sus Apócrifos (1970). Además de escribir estos libros, Pablo escribió y publicó muchas otros trabajos a lo largo de su vida, y colaboró con diferentes revistas pedagógicas y a través artículos de prensa. En 1967 Cobos fue rehabilitado como maestro, sin posibilidad de asumir cargos directivos. Fue entonces cuando trabajó en la escuela Asunción Nuestra Señora hasta 1969, cuando se jubila. Cobos falleció el 3 de enero de 1973 en Madrid.

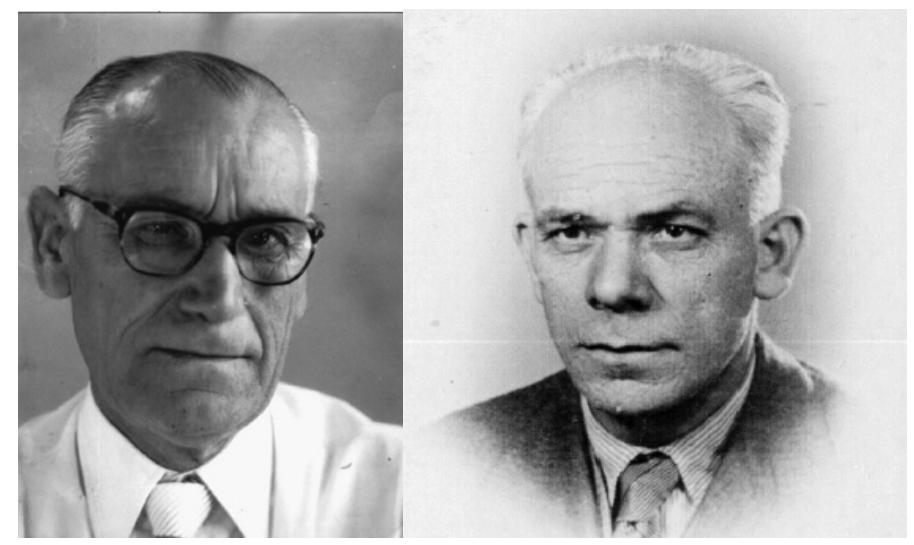

Figura 4. Norberto Hernanz (a la izquierda) y Pablo de Andrés (a la derecha). Fuente: Mora y Hermida (2011). 


\section{David Bayón Carretero}

David Bayón nació en torno al año $1897^{15}$ en Segovia. En 1918 fue maestro de escuela en Riaza, municipio de Segovia ${ }^{16}$. En 1924 se le concedió una pensión en grupo, dirigida por el inspector Vicente Valls Anglés ${ }^{17}$, mediante la cual visitó escuelas primarias y otras instituciones educativas de Francia, Bélgica y Suiza (JAE, 1925), donde trabajó aspectos relacionados con la organización y los métodos de enseñanza (Marín, 1990). Esta experiencia tuvo que ser muy enriquecedora para Bayón, pues en 1925 volvió a solicitar una nueva ayuda de ampliación de estudios, esta vez convocada por la Diputación de Segovia para los "estudiantes pobres de la provincia" ${ }^{18}$, y a la que también postuló Cobos; no obstante, esta ayuda no se le concedió, ya que a pesar de haber presentado la instancia, por motivos desconocidos no entregó la memoria que exigía la convocatoria (ADPSg, caja 4007).

La instancia que entregó y que localizamos en este mismo lugar, nos permite conocer que en estos momentos era maestro en la escuela de niños número 2 de Cuéllar, CEIP La Villa en la actualidad. El 20 de marzo de ese mismo año, Bayón impulsa en este centro una Asociación de Amigos de la Escuela con la finalidad de conseguir que se construya un nuevo edificio para la escuela de niños (CEIP La Villa, 2018). Bayón fue el primer Director de estas nuevas instalaciones, que comprendían el grupo escolar graduado "La Villa", inauguradas en 1928, tal y como figura en la placa conmemorativa situada actualmente en el centro (Figura 5).

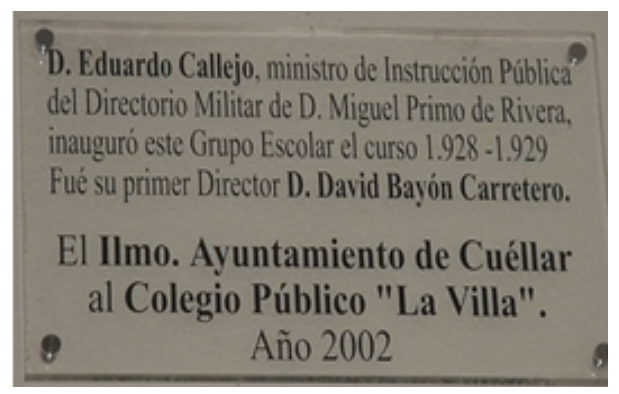

Figura 5. Placa conmemorativa de CEIP La Villa. Fuente: CEIP La Villa, 2018.

En 1927 volvió a solicitar una de las pensiones que otorgaba la Diputación de Segovia. Esta vez pudo disfrutar de una pensión de 1500 pesetas junto a Norberto Hernanz, Lucía Lucha García de la Llave y Francisca Gómez Gómez ${ }^{19}$. En compañía de Norberto visitó escuelas de Madrid, Zaragoza, Barcelona, Valencia, Granada y Huelva (Hernanz, s.f.). En 1930 volvió a recibir esta misma pensión para visitar escuelas del extranjero, esta vez con Norberto y Cobos (Dueñas y Grimau, 2009).

\footnotetext{
${ }^{15}$ Información recuperada de un árbol genealógico creado mediante la aplicación MyHeritage. Recuperado de https://goo.gl/BgUxT6

${ }^{16}$ Boletín Oficial de la Provincia de Segovia, 19 de febrero de 1923. Recuperado de https://goo.gl/cBDp5t (Archived by WebCite ${ }^{\circledR}$ at http://www.webcitation.org/6xYMzqPBH)

${ }^{17}$ El grupo estaba compuesto, además, por José Briones Martínez, Máximo Cajal Sarosa, Fausto de las Cuevas Díaz, Antonio Barcia Martín, Ángel Aniceto Gracia Morales, Esteban Granullaque Sánchez, Alberto López Casero y Edmundo Ruiz Yagüe (JAE, 1925).

${ }_{18}$ Nomenclatura que figura en las bases de la convocatoria de 1925 (ADPSg, caja 4007).

19 El Magisterio Español, 10 de agosto de 1927. Recuperado de https://goo.gl/Bfx7YQ (Archived by WebCite ${ }^{\circledR}$ at http://www.webcitation.org/6xYPVstci)
} 
En 1929 postuló a la escuela graduada de niños número 1 de Segovia, puesto que ocupó Hernanz, quedando él en el segundo lugar ${ }^{20}$. Al igual que Hernanz y Cobos, en 1931 se trasladó a Barcelona tras haber conseguido una vacante del Patronato Escolar (Andrés, 1970) ${ }^{21}$. En 1932 fue convocado para ocupar la sección de niños de la escuela situada en el número 22 de la avenida de La Inmaculada (Madrid) ${ }^{22}$. Este nombramiento se anuló y se le adjudicó la sección de niños, situada en el número 1 de la calle Don Pedro de Madrid ${ }^{23}$.

En 1934 publica junto al maestro Ángel Ledesma El método de proyectos Realizaciones. Esta obra y el artículo que publicó en Escuelas de España (Bayón, enero, 1934), "Fundamentos del método de proyectos", evidencian el interés que el maestro tenía por este método y por su impulsor, John Dewey, a quien hace continuas referencias. En ambos escritos relaciona el método con la filosofía y con la teoría y los principios que le otorgan sentido, haciendo especial hincapié en el valor del "hacer" en la escuela para lograr fines con una significación educativa y social. Asimismo, incluye modelos y orientaciones para llevar a cabo este tipo de métodos activos, heredados de las corrientes pedagógicas europeas.

Desconocemos en qué medida le afectó a este maestro la represión franquista. No obstante, Bayón fue uno de los veinte maestros de Valladolid que fue dado de baja en la escuela nacional en la que ejercía como docente, siguiendo lo dispuesto en la Orden de la Junta de Defensa Nacional de España del 19 de agosto de $1936^{24}$. Sabemos que finalizó su labor como maestro en 1961, jubilándose en el grupo escolar Villalpando (Segovia), del que fue su director (Matesanz, 2006).

\section{Un proyecto pedagógico compartido: la revista Escuelas de España}

Cobos, Bayón y Hernanz tuvieron una relación tanto profesional, como de amistad, llegando a ser conocidos como "los tres mosqueteros" ${ }^{25}$ (Hernanz, 2008). El viaje que realizaron Hernanz y Bayón por las escuelas de España, y que posteriormente hicieron a las escuelas del extranjero, esta vez acompañados por Cobos, hizo que compartiesen importantes experiencias y pudieran intercambiar impresiones. Hernanz (2008) cuenta que a veces, como era normal, tenían discrepancias, especialmente Bayón y Cobos. Hernanz (s.f.), que actuaba como mediador, nos da a conocer aspectos de la personalidad de los tres maestros:

\footnotetext{
${ }^{20}$ El Magisterio Español, 11 de julio de 1929. Recuperado de https://goo.gl/opPUir (Archived by WebCite ${ }^{\circledR}$ at http://www.webcitation.org/6xYPfp68W)

${ }^{21}$ Se anunció en la prensa de numerosos lugares de España. Algunos ejemplos son: Heraldo de Zamora, 13 de febrero de 1931. Recuperado de https://goo.gl/XEwuD3 (Archived by WebCite ${ }^{\circledR}$ at http://www.webcitation.org/6xYPqecxG); La Federación Escolar, 13 de febrero de 1931. Recuperado de https://goo.gl/XsuoQQ (Archived by WebCite ${ }^{\circledR}$ at http://www.webcitation.org/6xYQ1/tYD); Correo Extremeño, 14 de febrero de 1931. Recuperado de https://goo.gl/Fxdr1G (Archived by WebCite ${ }^{\circledR}$ at http://www.webcitation.org/6xYQBHhWc)

${ }^{22}$ Orden nombrando definitivamente a D. David Bayón Carretero Maestro propietario de la Sección de niños de nueva creación de la avenida de la Inmaculada, número 22, Madrid. La Gaceta de Madrid, 30 de junio de 1932. Recuperado de https://goo.gl/F1LUQv (Archived by WebCite ${ }^{\circledR}$ at http://www.webcitation.org/6xYQQx0hn)

${ }^{23}$ El Magisterio Español, 13 de agosto de 1932. Recuperado de https://goo.gl/jGnjgA (Archived by WebCite ${ }^{\circledR}$ at http://www.webcitation.org/6xYQaPPua)

${ }_{24}$ El Día de Palencia, 14 de septiembre de 1936. Recuperado de https://goo.gl/PuZMfF (Archived by WebCite ${ }^{\circledR}$ at http://www.webcitation.org/6xYQjUcRa)

${ }^{25}$ Denominación que después tomaron los maestros Pedro Natalías, Julio Fuster y Ángel Gracia, "... firmando sus escritos con los nombres mosqueteriles de Porthos, Athos y Aramis" (p. 127). Los tres fueron fusilados con el inicio de la Guerra Civil.
} 
Bayón, bajito, retrepado, concienzudo, realista, con frecuencia irónico y a veces mordaz, se mostraba sin reparos siempre dispuesto a criticar los juicios, un tanto precipitados, de su compañero Cobos. Este, un tanto fanfarrón y amigo de sentencias rotundas, se sentía herido por las observaciones crudamente realistas de su compañero Bayón. [...] Yo tuve que intervenir, conciliador, para evitarlo, pues creo que se me concedía alguna autoridad, tal vez porque, en relación a ambos, yo era tímido, sentimental, indeciso y un poco masoquista. No obstante, alguna vez fui también víctima de las ironías y burlas crueles de Bayón. (p.142)

En la carta que recoge Mora y Hermida (2011), y que Norberto envía a Cobos desde Barcelona el 2 de diciembre de 1934, también se aprecia este aspecto

A ver qué hacéis y tenerme al corriente de lo que dispongáis. De todos modos, no dejes a Bayón solo. Aunque riñáis alguna vez, esto es preferible a que tengáis reservas que entre nosotros no debe haber ya de ningún modo. Nos debemos a una obra y a ella hay que sacrificar todo; el amor propio, el éxito individual, la tendencia política o pedagógica, quedando a salvo el decoro moral únicamente. (p.105)

La obra a la que se refiere Norberto es uno de los proyectos que los tres maestros abordaron juntos: la revista pedagógica Escuelas de España. Está compuesta por 44 números que se publicaron entre enero de 1929 y julio de 1936 (excepto entre enero de 1932 y enero de 1934, que la revista estuvo inactiva por cuestiones económicas). Esta revista nació de la necesidad de perfeccionamiento que tenían los fundadores y del deseo de transformar la educación y las escuelas del momento, así como del grato funcionamiento de los tres congresos pedagógicos provinciales que se habían llevado a cabo en 1927, en 1928 y en 1929, y de los que ellos habían formado parte. Era necesario, por tanto, reflejar y dar a conocer las iniciativas educativas que se estaban llevando a cabo en el contexto español. Y qué mejor manera que fundar una revista dirigida al magisterio primario en la que escribieron profesionales de la educación de gran influencia en la época ${ }^{26}$ (Lozano, 1980). Ideológicamente, estaba vinculada a la Federación de Trabajadores de la Enseñanza (FETE-UGT), identificándose con los maestros que tenían un pensamiento educativo más liberal y renovador (Checa, 2002).

Esta fue, sin duda, la obra que más les unió. Cobos y Hernanz mantuvieron contacto hasta los últimos años de vida del primero; en la carta que Cobos envía tras salir de prisión a Hernanz, y en las sucesivas (Mora y Hermida, 2011), se observan continuas muestras de afecto -"yo sé muy bien que me quieres entrañablemente, que me quieres tanto como yo a ti [...] yo sé que somos de veras hermanos y lo hubiera seguido sabiendo aunque hubieras guardado silencio hasta el día de la muerte"- ( $p$. 106), además de anhelos del proyecto que juntos acometieron -"yo pensaré siempre que renace Escuelas de España" (p. 109)-, valoraciones de los más conocidos pensadores $^{27}$, o reflexiones sobre otras vivencias que habían compartido, como el traslado a Barcelona en 1931: "Tu pereza, aguantando Barcelona, fue un error. [...] Acaso no fue error el ir los tres a Barcelona, que nos deparó una experiencia fecunda; sí lo fue quedarte" (p. 140).

\section{Discusión}

La significación y la relevancia científica que tiene este trabajo se complementa con una importante significación humana. A través de este proyecto, como ya se viene haciendo con otros maestros desde las aportaciones reflejadas en la revisión de la

\footnotetext{
${ }^{26}$ Manuel Bartolomé Cossío, Ángel Llorca i García, Antonio Ballesteros Usano, Concepción Sáinz-Amor, Justa Freire Méndez, Félix Martí Alpera o Luis Álvarez Santullano, entre otros.

${ }_{27}$ José Ortega y Gasset, Xavier Zubiri Apalategi, Manuel Bartolomé Cossío o Antonio Machado Ruiz, entre otros.
} 
literatura, se pretende que los nombres de los maestros de escuela que trabajaron para mejorar la educación en un momento determinado no se borren de la historia. Concretamente, se contribuye a la recuperación individual y conjunta de tres personas, vinculándolas a las iniciativas educativas y de renovación pedagógica más importantes que protagonizaron. Todo ello se ha realizado mediante el análisis de un conjunto de trabajos científicos ya publicados, así como de fuentes primarias que nos acercan a cada uno de los docentes.

Su figura y actividad educativa queda vinculada a iniciativas representativas de la educación republicana, como lo son los CCP, los congresos pedagógicos provinciales, la realización y participación en Sociedades de Amigos de la Escuela y en Sociedades Infantiles, la colaboración en Misiones Pedagógicas, la creación de cantinas y de roperos en los centros educativos y la realización de viajes por escuelas ejemplares de España y del extranjero mediante las pensiones de la JAE y de otras Instituciones, así como la posterior puesta en práctica y transmisión de lo aprendido en conferencias, en artículos de prensa y en libros. Son, por tanto, figuras esenciales en el proceso de reconstrucción de la realidad educativa del primer tercio del siglo XX en España. Tal y como indican Mora y Hermida (2011), estudios de esta índole, como la recopilación de correspondencia que ellos realizan,

... nos permiten acceder al alma de estos maestros, perdedores de la guerra civil, pero defensores de un espíritu, heredado de otros que fueron a su vez maestros y construido por ellos mismos de manera reflexiva en las escuelas de cualquier rincón [...] o en empresas intelectuales como publicaciones, agrupaciones, instituciones... (p. 102)

De igual modo, Pozo (2013) señala que Hernanz, Bayón y Cobos pertenecieron a un grupo de maestros “... reflexivos con su propia práctica [...] [a quienes] se les cercenó su vida profesional en el momento en el que estaban en la cúspide de sus carreras, en el instante estelar de mayor productividad pedagógica" (p. 12). El valor que se les concede, como se puede observar en muchos otros trabajos ya citados a lo largo de este documento, hace necesario un proyecto como este.

Además de sintetizar la información ya disponible en otras obras, tratamos de hacer hincapié en los puntos de convergencia que unían a los tres maestros. Por otra parte, se han completado los datos de David Bayón mediante la recuperación de información contenida en fuentes primarias no analizadas anteriormente, lo cual es una clara fortaleza del estudio. Por el contrario, la ausencia de datos biográficos y de la vida de este maestro que nos permitan esclarecer un poco más su trayectoria, constituye una de las limitaciones de esta investigación, al mismo tiempo que conforma una oportunidad de continuidad del trabajo. En este sentido, la triangulación de la información que se establece en relación a los datos de Hernanz y Cobos, por provenir de fuentes diversas, constituye otra de las fortalezas del artículo, mediante el cual hemos pretendido unir las piezas de un puzle que representa una misma realidad y que nos lleva a alcanzar el objetivo que nos hemos propuesto: recuperar la figura de los maestros Norberto Hernanz Hernanz, David Bayón Carretero y Pablo de Andrés Cobos, además de contribuir a la producción científica de artículos de esta misma índole, con la finalidad de dar a los maestros y a las maestras el espacio, el reconocimiento y la dedicación que merecen dentro de la historia de la educación. 


\section{Referencias}

ADPSg. (1925). Caja 4007. Archivo de la Diputación Provincial de Segovia.

Agulló, MC. y Romans, V. (2018). 20 mestres del segle XX al País Valencià. Valencia: Edicions96.

Alonso, R. (2009). Concepción Sáinz-Amor: burgalesa, maestra y pionera. Brotes, 6, 13-16. (Archived by WebCite ${ }^{\circledR}$ at http://www.webcitation.org/6xYNAAu4D)

Andrés de, P. (octubre, 1931). Glosando las bases de La Granja. Escuelas de España, 16-25. (Archived by WebCite ${ }^{\circledR}$ at http://www.webcitation.org/6xYNVZU2b)

Andrés de, P. (1970). Juicios y figuras. Madrid: Ancos.

Andrés de, P., Zufriategui, A. y Estebaranz, A. (2015). La hora de la aldea. Navarra: Gráficas Castuera.

Andrés de, P., Zufriategui, A. y Estebaranz, A. (2017). El maestro, la escuela y la aldea y otros escritos pedagógicos. Segovia: Ayuntamiento de Segovia.

Andrés de, S. y Mora, JL. (2011). De ley y de corazón. Historia epistolar de una amistad. María Zambrano Alarcón - Pablo de Andrés Cobos - Cartas (1957-1976). Madrid: Servicio de Publicaciones de la Universidad Autónoma de Madrid.

Bayón, D. (enero, 1934). Fundamentos del método de proyectos. Escuelas de España, 19-27. (Archived by WebCite ${ }^{\circledR}$ at http://www.webcitation.org/6xYNey6VN)

CEIP La Villa. (2018). Nuestra historia. (Archived by WebCite ${ }^{\circledR}$ at http://www.webcitation.org/6xYNrrgo3)

Checa, A. (2002). Historia de la prensa pedagógica en España. Sevilla: Universidad de Sevilla.

Corbetta, P. (2003). Metodología y técnicas de investigación social. Madrid: McGraw-Hill.

Cossio, MB. (abril, 1929). Escuelas y escuela. Escuelas de España, 2, 3-6. (Archived by WebCite ${ }^{\circledR}$ at http://www.webcitation.org/6xYO50VUz)

Cuenca, A. (2003). La obra de Elisa López Velasco. Arte, Individuo y Sociedad, (15), 73-81.

Dueñas de, C. y Grimau, L. (2004). La represión franquista de la enseñanza en Segovia. Valladolid: Ámbito.

Dueñas de, C. y Grimau, L. (2009). De las sombras a la luz. La educación en Segovia (1900-1931). Madrid: Talasa Ediciones S.L.

Estebaranz, A. (1, marzo, 2016). Pablo de Andrés Cobos: maestro en La Granja. Arqueología de Imágenes [Blog]. (Archived by WebCite ${ }^{\circledR}$ at http://www.webcitation.org/6xYODLLtm)

García, N. (2008). La recuperación de Norberto Hernanz Hernanz (Maestro segoviano, fundador de los Centros de Colaboración Pedagógica). El Adelantado de Segovia. (Archived by WebCite ${ }^{\circledR}$ at http://www.webcitation.org/6xYOQfDYZ)
González, T. (2009). Educación y fronteras. María Sánchez Arbós y su lucha por la renovación pedagógica en Canarias (19201925). Revista de Educación, 356, 431-455.

Hermida, F. (2005). Pablo de Andrés Cobos. En J. L. Mora y J. M. Moreno (Coords.), Pensamiento y palabra en recuerdo de María Zambrano (1904-1991): contribución de Segovia a su empresa intelectual (pp. 337-362). Valladolid: Junta de Castilla y León, Consejería de Cultura y Turismo.

Hernández, JC. (2007). Los maestros de la República. Memoria y olvido en tiempos de libertad. Foro de Educación, 9, 153-168.

Hernanz, N. (enero, 1929). Formación del magisterio. Escuelas de España, 1, 6-27. (Archived by WebCite ${ }^{\circledR}$ at http://www.webcitation.org/6xYOWkgsA)

Hernanz, M. (2008). Notas sobre la vida profesional de un maestro de escuela segoviano (1891-1939). Manuscrito inédito.

Hernanz, N. (s.f.). Memorias Norberto Hernanz y Hernanz. Manuscrito inédito.

Iglesias, MA. (2006). Maestros de la República: los otros santos, los otros mártires. Madrid: La Esfera de los Libros.

JAE. (1925). Memoria correspondiente a los cursos 1922-3 y 1923-4. Madrid: JAE. (Archived by WebCite ${ }^{\circledR}$ at http://www.webcitation.org/6xYOcwz9f)

Jiménez, JA. (1992). Contribuciones de Santiago Hernández Ruiz al proceso de modernización y renovación escolar en España (1901-1998). Historia de la educación: Revista interuniversitaria, 11, 287-296.

Jimeno, C. (2009). María Sánchez Arbós (1889-1976) el exilio interior de una maestra. Iber: Didáctica de las Ciencias Sociales, Geografía e Historia, 62, 46-55.

Juan, VM. (1999). María Sánchez Arbós. Una maestra aragonesa en la edad de oro de la Pedagogía. Rolde. Revista de Cultura Aragonesa, 89, 12-21.

Juan, VM. (2017). Crónicas de la vieja pizarra. Escuelas y maestros: semblanzas históricas de la educación en Aragón. Zaragoza: Doce Robles.

Lázaro, LM. (2009). Luis Huerta: eugenesia, medicina y pedagogía en España. Historia de la Educación: Revista Interuniversitaria, 28, 61-88.

Llopis, R. (2005). La revolución en la escuela (Antonio Molero, Ed.). Madrid: Biblioteca Nueva.

López, C. (1995). Ángel Llorca: un educador actual. Docencia e Investigación: Revista de la Escuela Universitaria de Magisterio de Toledo, 5, 101-116.

Lozano, C. (1980). La educación republicana 1931-1939. Barcelona: Universitat de Barcelona.

MacDonald, K., \& Tipton, C. (1993). Using documents. In N. Gilbert (Ed.), Researching social life (pp. 187-200). London: Sage Ltd. 
Marín, T. (1990). La renovación pedagógica en España (19071936). Los pensionados en pedagogía por la Junta para la Ampliación de Estudios. Madrid: Artes Gráficas.

Marín, T. (1991). Innovadores de la educación en España (becarios de la Junta para la Ampliación de Estudios). Castilla - La Mancha: Servicio de Publicaciones de la Universidad de Castilla - La Mancha.

Martín, B. (2015). La cultura escolar y el oficio de maestro. Educación XX1: Revista de la Facultad de Educación, 18(1), 147-166.

Martínez, JA. (2000). La Segunda República (1931-1936). En A. Bahamonde (Coord.), Historia de España. Siglo XX (pp. 541636). Madrid: Ediciones Cátedra.

Matesanz, J. (2006). El centro visto por sus anteriores directores. Colegio Villalpando 50 aniversario, 8-9.

Molero, A. (1997). Los maestros republicanos: legislación y conflictividad profesional (1931-1936). Historia de la Educación: Revista Interuniversitaria, 16, 285-302.

Mora, JL. y Hermida, F. (2011). En torno a Ortega y Gasset, Machado y Zubiri. Epistolario: Norberto Hernanz - Pablo de A. Cobos (1934-1971). Revista de Hispanismo Filosófico, 16, 95-143.

Moreno, PL. (2005). Renovación pedagógica y compromiso social en la edad de plata de la Pedagogía española: Félix Martí Alpera (1898-1920). Revista Española de Pedagogía, 231, 203-222.

Montanyà, O. (2006). Los maestros de la República. Cambio, 1793, 22-23.

Navarro, C. (2002). La figura del maestro, en la Escuela de la República. Revista Interuniversitaria de Formación del Profesorado, 43, 21-37.

Ortiz, R. (2017). La renovación pedagógica desde la mirada de la revista Escuelas de España (1929-1931). Trabajo de Fin de Máster (Máster de investigación en ciencias sociales. Educación, comunicación audiovisual, economía y empresa). Universidad de Valladolid. (Archived by WebCite ${ }^{\circledR}$ at http://www.webcitation.org/6xYPEm9Aw)

Pozo del, MM. (1987). Ángel Llorca: un maestro entre la Institución Libre de Enseñanza y la Escuela Nueva (1866-1942). Historia de la Educación: Revista Interuniversitaria, 6, 229-248.

Pozo del, MM. (2012). La construcción de la categoría 'maestra republicana': la tipología generacional como propuesta. En E. Sánchez (Ed.), Las maestras de la República (pp. 236-270). Madrid: Catarata.

Pozo del, MM. (2013). Justa Freire o la pasión de educar. Biografía de una maestra atrapada en la historia de España (1896-1965). Barcelona: Octaedro.

Puelles de, M. (2011). Politica y educación en la España contemporánea. Madrid: Universidad Nacional de Educación a Distancia.

Ruiz, J. (1976). El método histórico en la investigación histórica de la educación. Revista Española de Pedagogía, 34(134), 449475 .
Ruiz, J. (1997). El método histórico en la investigación históricoeducativa. En A. Viñao, y M. Gabriel (Coords.), La investigación histórico-educativa: tendencias actuales (pp. 131-202). Barcelona: Editorial Ronsel.

Sánchez, CM. (2010). La figura de la maestra rural en la Segunda República. Revista de Antropología Experimental, 10, 119128.

Tiana, A. (1988). La investigación histórico-educativa actual. Enfoques y métodos. Madrid: Universidad Nacional de Educación a Distancia.

Vicente, J. (2006). República de maestros. Aula Libre, 84, 7-9.

Vilafranca, I. y Vilanou, C. (2001). Concepción Sainz-Amor: Viena, laboratorio de Pedagogía. Historia de la Educación: Revista Interuniversitaria, 20, 449-465. 